\title{
ERROR ANALYSIS FOR SPARSE TIME-FREQUENCY DECOMPOSITION OF SIGNALS BASED ON PROBABILITY
}

\author{
Qi Yang \\ Department of Mathematics, Jinan University, Guangzhou, 510632, P. R. China
}

\begin{abstract}
In this paper, after reviewing the data driven time-frequency analysis (DDTFA) and the matching pursuit method in decomposition of intrinsic mode functions (IMFs), we estimate the orthogonality properties between IMFs based on probability. By theoretical proofs and numerical experiments, we show that the estimation in [9] is the best.
\end{abstract}

Keywords: sparse time-frequency decomposition, scale separation, nonlinear matching pursuit.

\section{Introduction}

Time-frequency analysis for the signal can help us to further process the signal more easily, and we can have a better understanding of various complex phenomena. In recent years, the Joint Time-Frequency

\footnotetext{
${ }^{*}$ Corresponding author.

E-mail address: yangqi@stu2016.jnu.edu.cn (Qi Yang).
}

Copyright @ 2019 Scientific Advances Publishers 2010 Mathematics Subject Classification: 90C26.

Submitted by L. Rajendran.

Received March 22, 2019; Revised March 27, 2019 
Analysis (JTFA) method has become a powerful tool for analyzing nonlinear non-stationary data. Common time-frequency analysis methods include wavelet transform (WT) [1], Wigner-Ville distribution [2], and Empirical Mode Decomposition (EMD) [3].

Inspired by the EMD method and compressive sensing $[4,5,6]$, Hou and Shi proposed a data-driven time-frequency analysis method [7, 8]. The data-driven time-frequency analysis method based on the sparsest representation of multiscale data to decompose the signal into a finite number of IMF with a small residual:

$$
f(t)=\sum_{k=1}^{M} a_{k}(t) \cos \theta_{k}(t)+r(t), \quad t \in[0, T],
$$

where each IMF is a signal with amplitude modulation-frequency modulation (AM-FM). The amplitude $a_{k}(t)>0$, the instantaneous frequency $\omega_{k}(t)=\theta_{k}(t)>0$, and $r(t)$ is a small residual.

The idea of this method is looking for the sparsest representation over the dictionary consisting of all IMFs by solving the following nonlinear optimization problem:

$$
\begin{aligned}
& \underset{\left(a_{k}\right)_{1 \leq k \leq M,},\left(\theta_{k}\right)_{1 \leq k \leq M}}{\min _{1}} \\
& \text { subject to }\left|f(t)-\sum_{k=1}^{M} a_{k}(t) \cos \theta_{k}(t)\right| \leq \varepsilon, 0 \leq t \leq T, a_{k} \cos \theta_{k} \in D .\left(\mathrm{P}_{0}\right)
\end{aligned}
$$

The dictionary is defined as follow:

$$
D=\left\{a(t) \cos \theta(t): \theta^{\prime}(t) \geq 0, a(t) \in V(\theta)\right\},
$$

where $V(\theta)$ is a is the collection of all the function that are less oscillatory than $\cos \theta(t)$. Under the assumption of the scale separation, the uniqueness of the optimization problem $\left(\mathrm{P}_{0}\right)$ problem is analyzed in the [9]. The definitions of scale separation and well-separated signal as follows. 
Definition 1.1 (Scale separation [9]). A function $f(t)=a(t) \cos \theta(t)$ $(t \in[0, T])$ is said to satisfy a scale separation property with a separation factor $\varepsilon>0$, if $a(t)$ and $\theta(t)$ satisfy the following conditions:

$$
\begin{aligned}
& a(t) \in C^{1}(\mathbb{R}), \theta \in C^{2}(\mathbb{R}), \inf _{t \in[0, T]} \theta^{\prime}(t)>0, \\
& \frac{\sup _{t \in[0, T]} \theta^{\prime}(t)}{\inf _{t \in[0, T]} \theta^{\prime}(t)} \leq M^{\prime}<+\infty,\left|\frac{a^{\prime}(t)}{a(t) \theta^{\prime}(t)}\right| \leq \varepsilon,\left|\frac{\theta^{\prime \prime}(t)}{\left(\theta^{\prime}(t)\right)^{2}}\right| \leq \varepsilon, \quad \forall t \in[0, T] .
\end{aligned}
$$

Definition 1.2 (Well-separated signal [9]). A signal $f:[0, T] \rightarrow \mathbb{R}$ is said to be well separated with separation factor $\varepsilon$ and frequency ratio $\beta$ if it can be written as

$$
f(t)=\sum_{k=1}^{M} a_{k}(t) \cos \theta_{k}(t)+r(t),
$$

where all $f_{k}(t)=a_{k}(t) \cos \theta_{k}(t)$ satisfy the scale separation property with separation factor $\varepsilon, r(t)=O(\varepsilon)$, and their phase function $\theta_{k}(t)$ satisfy

$$
\theta_{k}^{\prime}(t) \geq \beta \theta_{k-1}^{\prime}(t), \quad \forall t \in[0, T],
$$

and $\beta>1, \beta-1=O(1)$.

Because the problem $\left(\mathrm{P}_{0}\right)$ is nonlinear and nonconvex, it is too difficult to solve. Based on matching pursuit [10], a nonlinear matching pursuit (NMP) is introduced. The NMP algorithm is as follows:

Task. To find an approximate optimizer of the problem $\left(\mathrm{P}_{0}\right)$.

Initialization: Set the initial residual $r_{0}=f$ and $k=1$.

\section{Main iteration:}

Step 1. Solve the nonlinear least-squares problem as follows:

$$
\begin{aligned}
& \min _{a_{k}, \theta_{k}}\left\|r_{k-1}-a_{k} \cos \theta_{k}\right\|_{L^{2}}^{2} \\
& \text { subject to } a_{k} \in V\left(\theta_{k}\right), \theta_{k}>0 .
\end{aligned}
$$


Step 2. Update the residual

$$
r_{k}=f-\sum_{j=1}^{k} a_{j} \cos \theta_{j}
$$

Step 3. If $\left\|r_{k}\right\|_{L^{2}}<\varepsilon_{0}$, stop. Otherwise, set $k=k+1$ and go back to Step 1 .

In this paper, we decompose $f$ over the dictionary

$$
D_{\varepsilon}:=\left\{a(\cdot) \cos \theta(\cdot):(a, \theta) \in U_{\varepsilon}\right\},
$$

where

$$
U_{\varepsilon}:=\left\{(a, \theta) ; a>0, \theta^{\prime}>0 ;\left|\frac{a^{\prime}}{a \theta^{\prime}}\right| \leq \varepsilon,\left|\frac{\theta^{\prime \prime}}{\left[\theta^{\prime}\right]^{2}}\right| \leq \varepsilon, \frac{\sup _{t \in[0, T]} \theta^{\prime}(t)}{\inf _{t \in[0, T]} \theta^{\prime}(t)} \leq M^{\prime}\right\} .
$$

The main idea of the NMP algorithm is solving the following nonlinear least-squares problem:

$$
\begin{aligned}
& \text { minimize } p(a, \theta):=\|f(t)-a(t) \cos \theta(t)\|_{L^{2}}^{2} \\
& \text { subject to }(a, \theta) \in U_{\varepsilon} .
\end{aligned}
$$

We consider the error of the sparse time-frequency decomposition of signals based on probability. By the proof of ([3], Theorem 3.1), we have the following result:

Lemma 1.3. Let $f(t)$ be a function satisfying the scale separation property with separation factor $\varepsilon$ and frequency ratio $\beta$ as defined in Definition 1.2:

$$
f(t)=\sum_{k=1}^{M} a_{k}(t) \cos \theta_{k}(t)+r(t), \quad a_{k} \cos \theta_{k} \in U_{\varepsilon}, \quad a_{k}=O(1), r=O(\varepsilon) .
$$


Suppose that $l \in\{1, \ldots, M\}$. If

$$
p(a, \theta) \leq p\left(a_{l}, \theta_{l}\right)
$$

where $p(a, \theta)$ is given in $\left(\mathrm{P}_{2}\right)$, then we have

$$
\frac{\left\|a \cos \theta-a_{l} \cos \theta_{l}\right\|_{L^{2}}}{\left\|a_{l} \cos \theta_{l}\right\|_{L^{2}}} \leq \delta_{2}+\sqrt{\delta_{2}^{2}+2\left(\delta_{1}+\delta_{2}\right)},
$$

where

$$
\delta_{1}=\sum_{k \neq l} \mu_{k, l} \frac{\left\|a_{k} \cos \theta_{k}\right\|_{L^{2}}}{\left\|a_{l} \cos \theta_{l}\right\|_{L^{2}}}, \mu_{k, l}=\frac{\left|\left\langle a_{l} \cos \theta_{l}, a_{k} \cos \theta_{k}\right\rangle\right|}{\left\|a_{l} \cos \theta_{l}\right\|_{L^{2}}\left\|a_{k} \cos \theta_{k}\right\|_{L^{2}}},
$$

and

$$
\delta_{2}=\sum_{k \neq l} \gamma_{k, l} \frac{\left\|a_{k} \cos \theta_{k}\right\|_{L^{2}}}{\left\|a_{l} \cos \theta_{l}\right\|_{L^{2}}}+\frac{\|r(t)\|_{L^{2}}}{\left\|a_{l} \cos \theta_{l}\right\|_{L^{2}}}, \gamma_{k, l}=\frac{\mid\left\langle a \cos \theta, a_{k} \cos \theta_{k}\right\rangle}{\|a \cos \theta\|_{L^{2}}\left\|a_{k} \cos \theta_{k}\right\|_{L^{2}}} .
$$

\section{Error Analysis Based on Probability}

In the actual information transmission process, the signal cannot arrive on time due to the long distance of transmission, such as the busy network or some faults in the transmission, which may result in phase delay of the signal. We introduce phase delay parameters and discuss $f$ on the time domain $[0, T]$.

From Lemma 1.3, we know that the error of the sparse timefrequency decomposition of the signal depends on the coherences between $a_{k} \cos \theta_{k}$ and $a_{l} \cos \theta_{l}$. By ([9], Corollary 3.3), we have

$$
\mu_{k, l}=\frac{\left|\left\langle a_{l} \cos \theta_{l}, a_{k} \cos \theta_{k}\right\rangle\right|}{\left\|a_{l} \cos \theta_{l}\right\|_{L^{2}}\left\|a_{k} \cos \theta_{k}\right\|_{L^{2}}}=O(\varepsilon),
$$

and the error between the IMF computed by NMP algorithm and the true IMF is $O(\sqrt{\varepsilon})$. In this section, we introduce random variables to discuss 
the error of signal sparse time-frequency decomposition from the perspective of probability. We assume that the phase delay parameter is a uniformly distributed random variable obeying $[0,2 \pi]$. Then we have

$$
f(t)=\sum_{k=1}^{M} a_{k}(t) \cos \left(\theta_{k}(t)+X_{k}\right)
$$

where $X_{k} \sim U[0,2 \pi], k \in\{1,2, \ldots, M\}$. We define functions

$$
\begin{aligned}
g\left(X_{k}, X_{l}\right) & :=\left\langle a_{k} \cos \left(\theta_{k}+X_{k}\right), a_{l} \cos \left(\theta_{l}+X_{l}\right)\right\rangle \\
& =\int_{0}^{T} a_{k}(t) \cos \left(\theta_{k}(t)+x_{k}\right) a_{l}(t) \cos \left(\theta_{l}(t)+x_{l}\right) d t,
\end{aligned}
$$

for $k \neq l \in\{1,2, \ldots, M\}$. We have the following theorem.

Theorem 2.1. Let $f(t)$ be a function satisfying the scale separation property with separation factor $\varepsilon$ and frequency ratio $\beta$ as defined in Definition 1.2:

$$
f(t)=\sum_{k=1}^{M} a_{k}(t) \cos \left(\theta_{k}(t)+X_{k}\right), \quad a_{k}=O(1), \forall t \in[0, T],
$$

where $\left(a_{k}, \theta_{k}\right)_{1 \leq k \leq M} \in U_{\varepsilon}$, Phase delay variable $X_{k}, X_{l} \sim U[0,2 \pi]$ $(k \neq l)$. Then we have

$$
\begin{gathered}
E\left[g\left(X_{k}, X_{l}\right)\right]=0 \\
D\left[g\left(X_{k}, X_{l}\right)\right]=\frac{1}{4} \int_{0}^{T} \int_{0}^{T} a_{k}\left(t_{1}\right) a_{l}\left(t_{1}\right) a_{k}\left(t_{2}\right) a_{l}\left(t_{2}\right) \\
\cdot \cos \left(\theta_{k}\left(t_{1}\right)-\theta_{k}\left(t_{2}\right)\right) \cos \left(\theta_{l}\left(t_{1}\right)-\theta_{l}\left(t_{2}\right)\right) d t_{1} d t_{2} .
\end{gathered}
$$


Proof. First, we have

$E\left[g\left(X_{k}, X_{l}\right)\right]$

$$
\begin{aligned}
& =\frac{1}{2 \pi} \cdot \frac{1}{2 \pi} \int_{0}^{2 \pi} \int_{0}^{2 \pi} d x_{k} d x_{l} \int_{0}^{T} a_{k}(t) \cos \left(\theta_{k}(t)+x_{k}\right) a_{l}(t) \cos \left(\theta_{l}(t)+x_{l}\right) d t \\
& =\frac{1}{4 \pi^{2}} \int_{0}^{T} a_{k}(t) a_{l}(t) d t \int_{0}^{2 \pi} \cos \left(\theta_{l}(t)+x_{k}\right) d x_{k} \int_{0}^{2 \pi} \cos \left(\theta_{l}(t)+x_{l}\right) d x_{l} \\
& =0 .
\end{aligned}
$$

Now, we calculate $D\left[g\left(X_{k}, X_{l}\right)\right]$.

$$
\begin{aligned}
& D\left[g\left(X_{k}, X_{l}\right)\right] \\
= & \frac{1}{2 \pi} \cdot \frac{1}{2 \pi} \int_{0}^{2 \pi} \int_{0}^{2 \pi} d x_{k} d x_{l}\left[\int_{0}^{T} a_{k}(t) \cos \left(\theta_{k}(t)+x_{k}\right) a_{l}(t) \cos \left(\theta_{l}(t)+x_{l}\right) d t\right]^{2} \\
= & \frac{1}{4 \pi^{2}} \int_{0}^{2 \pi} \int_{0}^{2 \pi} d x_{k} d x_{l}\left(\int_{0}^{T} a_{k}\left(t_{1}\right) \cos \left(\theta_{k}\left(t_{1}\right)+x_{k}\right) a_{l}\left(t_{1}\right) \cos \left(\theta_{l}\left(t_{1}\right)+x_{l}\right) d t_{1}\right) \\
& \left(\int_{0}^{T} a_{k}\left(t_{2}\right) \cos \left(\theta_{k}\left(t_{2}\right)+x_{k}\right) a_{l}\left(t_{2}\right) \cos \left(\theta_{l}\left(t_{2}\right)+x_{l}\right) d t_{2}\right)
\end{aligned}
$$

Since,

$$
\begin{aligned}
\int_{0}^{2 \pi} \cos \left(\theta_{k}\left(t_{1}\right)+x_{k}\right) \cos \left(\theta_{k}\left(t_{2}\right)+x_{k}\right) d x_{k} \\
\quad=\int_{0}^{2 \pi} \frac{1}{2}\left[\cos \left(\theta_{k}\left(t_{1}\right)+\theta_{k}\left(t_{2}\right)+2 x_{k}\right)+\cos \left(\theta_{k}\left(t_{1}\right)-\theta_{k}\left(t_{2}\right)\right)\right] d x_{k} \\
=\pi \cos \left(\theta_{k}\left(t_{1}\right)-\theta_{k}\left(t_{2}\right)\right),
\end{aligned}
$$

and similarly,

$$
\int_{0}^{2 \pi} \cos \left(\theta_{k}\left(t_{1}\right)+x_{k}\right) \cos \left(\theta_{k}\left(t_{2}\right)+x_{k}\right) d x_{k}=\pi \cos \left(\theta_{k}\left(t_{1}\right)-\theta_{k}\left(t_{2}\right)\right)
$$

we obtain (20) by exchange the integration order in (22). This completes the proof. 
Noticing that

$$
\begin{aligned}
D\left[g\left(X_{k}, X_{l}\right)\right]= & \frac{1}{4}\left[\left(\int_{0}^{T} a_{k}\left(t_{1}\right) a_{l}\left(t_{1}\right) \cos \theta_{k}\left(t_{1}\right) \cos \theta_{l}\left(t_{1}\right) d t_{1}\right)\right. \\
& \cdot\left(\int_{0}^{T} a_{k}\left(t_{2}\right) a_{l}\left(t_{2}\right) \cos \theta_{k}\left(t_{2}\right) \cos \theta_{l}\left(t_{2}\right) d t_{2}\right) \\
& +\left(\int_{0}^{T} a_{k}\left(t_{1}\right) a_{l}\left(t_{1}\right) \cos \theta_{k}\left(t_{1}\right) \sin \theta_{l}\left(t_{1}\right) d t_{1}\right) \\
& \cdot\left(\int_{0}^{T} a_{k}\left(t_{2}\right) a_{l}\left(t_{2}\right) \cos \theta_{k}\left(t_{2}\right) \sin \theta_{l}\left(t_{2}\right) d t_{2}\right) \\
& +\left(\int_{0}^{T} a_{k}\left(t_{1}\right) a_{l}\left(t_{1}\right) \cos \theta_{l}\left(t_{1}\right) \sin \theta_{k}\left(t_{1}\right) d t_{1}\right) \\
& \cdot\left(\int_{0}^{T} a_{k}\left(t_{2}\right) a_{l}\left(t_{2}\right) \cos \theta_{l}\left(t_{2}\right) \sin \theta_{k}\left(t_{2}\right) d t_{2}\right) \\
& +\left(\int_{0}^{T} a_{k}\left(t_{1}\right) a_{l}\left(t_{1}\right) \sin \theta_{k}\left(t_{1}\right) \sin \theta_{l}\left(t_{1}\right) d t_{1}\right) \\
& \left.\cdot\left(\int_{0}^{T} a_{k}\left(t_{2}\right) a_{l}\left(t_{2}\right) \sin \theta_{k}\left(t_{2}\right) \sin \theta_{l}\left(t_{2}\right) d t_{2}\right)\right]
\end{aligned}
$$

by ([9], Lemma 3.2), it is easy to check that

$$
\begin{aligned}
\left|\int_{0}^{T} a_{k}\left(t_{1}\right) a_{l}\left(t_{1}\right) \cos \theta_{k}\left(t_{1}\right) \cos \theta_{l}\left(t_{1}\right) d t_{1}\right| \\
=\left|\frac{1}{2} \int_{0}^{T} a_{k}\left(t_{1}\right) a_{l}\left(t_{1}\right) \cdot\left[\cos \left(\theta_{k}+\theta_{l}\right)+\cos \left(\theta_{k}-\theta_{l}\right)\right] d t_{1}\right| \\
=O(\varepsilon) .
\end{aligned}
$$

Other items in (24) have similar estimates. Thus, we get

$$
D\left[g\left(X_{k}, X_{l}\right)\right]=O\left(\varepsilon^{2}\right) .
$$


Is this estimate the best? Let us study this issue by an instance.

Example 2.2. Let us consider the two IMFs which is defined as following:

$$
i m f_{k}=a_{k} \cos \theta_{k}(t), i m f_{l}=a_{l} \cos \theta_{l}(t), \quad t \in[0,1],
$$

where

$$
\begin{aligned}
& a_{k}=c_{k}+\sum_{i=1}^{\lambda c_{k}}\left[a_{i k} \cos \left(\frac{\pi i}{T} t\right)+b_{i k} \sin \left(\frac{\pi i}{T} t\right)\right], \\
& a_{l}=c_{l}+\sum_{j=1}^{\lambda c_{l}}\left[a_{i l} \cos \left(\frac{\pi j}{T} t\right)+b_{i l} \sin \left(\frac{\pi j}{T} t\right)\right], \\
& \theta_{k}=8 \pi c_{k} t+\sum_{i=1}^{\lambda c_{k}}\left[a_{i k} \cos \left(\frac{4 \pi i}{T} t\right)+b_{i k} \sin \left(\frac{4 \pi i}{T} t\right)\right], \\
& \theta_{l}=7.5 \pi c_{l} t+\sum_{j=1}^{\lambda c_{l}}\left[a_{i l} \cos \left(\frac{3.5 \pi j}{T} t\right)+b_{i l} \sin \left(\frac{3.5 \pi j}{T} t\right)\right],
\end{aligned}
$$

and $c_{k}=8, c_{l}=6, \lambda=0.5, T=1 ; a_{i k}, b_{i k}, a_{i l}, b_{i l}\left(i=1, \ldots, \lambda c_{k}, j=1, \ldots, \lambda_{c_{l}}\right)$ are random number from -1 to 1 such that $\varepsilon_{0} \leq 0.1$, where

$$
\varepsilon_{0}=\max _{t \in[0,1]}\left\{\left|\frac{a_{k}^{\prime}(t)}{a_{k}^{\prime}(t) \theta_{k}^{\prime}(t)}\right|,\left|\frac{\theta_{k}^{\prime \prime}(t)}{\left[\theta_{k}^{\prime}(t)\right]^{2}}\right|,\left|\frac{a_{l}^{\prime}(t)}{a_{l}^{\prime}(t) \theta_{l}^{\prime}(t)}\right|,\left|\frac{\theta_{l}^{\prime}(t)}{\left[\theta_{l}^{\prime}(t)\right]^{2}}\right|\right\} .
$$

We present numerical experiments to show the relationship between $D\left[g\left(X_{k}, X_{l}\right)\right]$ and $\varepsilon_{0}^{2}$ in Example 2.2. We take 65000 different values for random numbers $a_{i k}, b_{i k}, a_{j l}, b_{j l}$, respectively and obtain the relationship between $D\left[g\left(X_{k}, X_{l}\right)\right] /\left(k_{0} \varepsilon_{0}^{2}\right)$ and $\varepsilon_{0}^{2}$ in Figure 1 , where

$$
k_{0}=\left(\left\|a_{l} \cos \theta_{l}\right\|_{L^{2}} \cdot\left\|a_{k} \cos \theta_{k}\right\|_{L^{2}}\right)^{2} .
$$




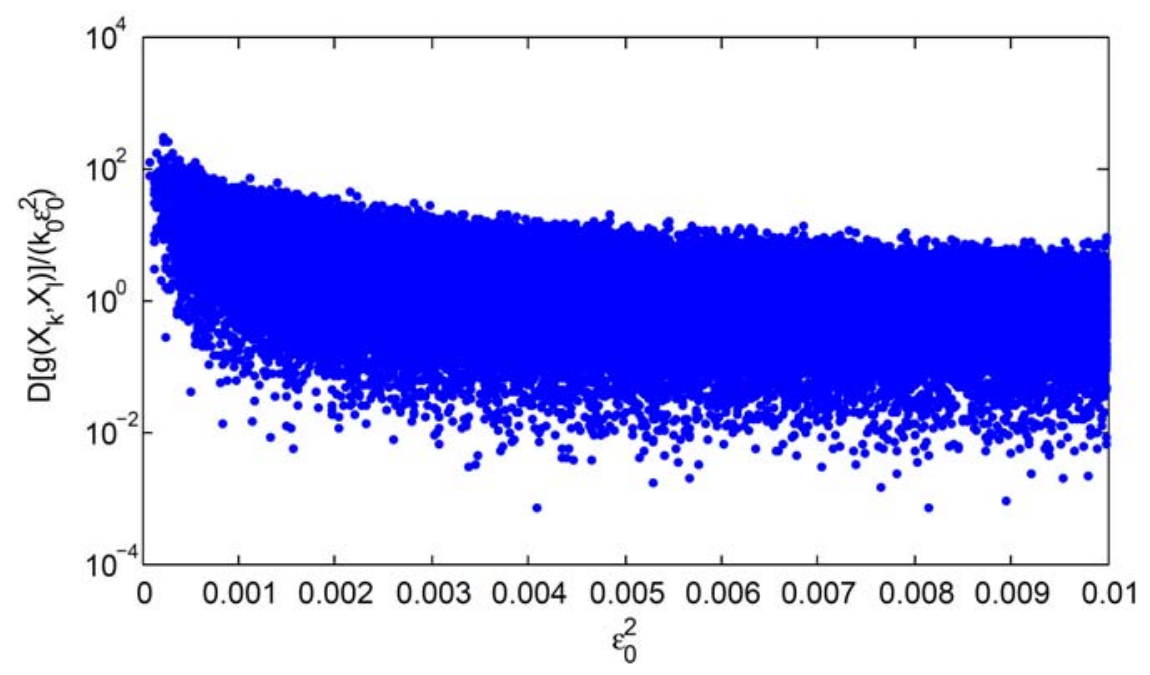

Figure 1. The scatter plot of $D\left[g\left(X_{k}, X_{l}\right)\right] /\left(k_{0} \varepsilon_{0}^{2}\right)$ and $\varepsilon_{0}^{2}$.

It can be seen from Figure 1 that, in Example 2.2, there exist two positive constants $M_{1}$ and $M_{2}$ such that

$$
M_{1} \leq\left|D\left[g\left(X_{k}, X_{l}\right)\right] /\left(k \varepsilon_{0}^{2}\right)\right| \leq M_{2} .
$$

In Example 2.2, we know that the estimation result in (15) already be the best estimate. But in Theorem 2.1, we have $E\left[g\left(X_{k}, X_{l}\right)\right]=0$, from the perspective of probability, $\mu_{k, l}$ may be smaller than that estimated in Equation (15). And the error between the computed IMF and the true IMF may be smaller than $O(\sqrt{\varepsilon})$.

\section{Concluding Remarks}

In this paper, based on probability, we consider the sparse timefrequency decomposition of signals by nonlinear matching pursuit method and analyze the error of finding a sparse representation of signals. By the theoretical proof, we show the mean and variance of the inner product between IMFs. And we present numerical experiments and study the relationship between the variance and the separation factor $\varepsilon$. 


\section{References}

[1] S. Mallat, A Wavelet Tour of Signal Processing: The Sparse Way, 3rd Edition, Elsevier/Academic Press, Amsterdam, 2009.

[2] P. Flandrin, Time-Frequency/Time-Scale Analysis, Academic Press, San Diego, 1999.

[3] N. E. Huang, Z. Shen, S. R. Long, M. C. Wu, H. H. Shih, Q. Zheng, N.-C. Yen, C. C. Tung and H. H. Liu, The empirical mode decomposition and the Hilbert spectrum for nonlinear and non-stationary time series analysis, Proc. R. Soc. Lond. Ser. A: Math. Phys. Eng. Sci. 454(1971) (1998), 903-995.

DOI: https://doi.org/10.1098/rspa.1998.0193

[4] D. L. Donoho, Compressed sensing, IEEE Transactions on Information Theory 52(4) (2006), 1289-1306.

DOI: https://doi.org/10.1109/TIT.2006.871582

[5] D. Gabor, Theory of communication, Journal of IEE 93(2) (1946), 429-457.

[6] E. J. Candès, J. Romberg and T. Tao, Robust uncertainty principles: Exact signal reconstruction from highly incomplete frequency information, IEEE Transactions on Information Theory 52(2) (2006), 489-509.

DOI: https://doi.org/10.1109/TIT.2005.862083

[7] T. Y. Hou and Z. Q. Shi, Adaptive data analysis via sparse time-frequency representation, Advances in Adaptive Data Analysis 3(1-2) (2011), 1-28.

DOI: https://doi.org/10.1142/S1793536911000647

[8] T. Y. Hou and Z. Q. Shi, Data-driven time-frequency analysis, Applied and Computational Harmonic Analysis 35(2) (2013), 284-308.

DOI: https://doi.org/10.1016/j.acha.2012.10.001

[9] C. G. Liu, Z. Q. Shi and T. Y. Hou, On the uniqueness of sparse time-frequency representation of multiscale data, Multiscale Modeling \& Simulation 13(3) (2015), 790-811.

DOI: https://doi.org/10.1137/141002098

[10] S. Mallat and Z. Zhang, Matching pursuits with time-frequency dictionaries, IEEE Transactions on Signal Processing 41(12) (1993), 3397-3415.

DOI: https://doi.org/10.1109/78.258082 